\title{
Nyctiphanes couchii as intermediate host for Rhadinorhynchus sp. (Acanthocephala, Echinorhynchidae) from NW Iberian Peninsula waters
}

\author{
M. Gregori ${ }^{1}$, F. J. Aznar ${ }^{2}$, E. Abollo ${ }^{3}$, A. Roura ${ }^{1}$, A. F. González ${ }^{1}$, S. Pascuall ${ }^{1, *}$ \\ ${ }^{1}$ Instituto de Investigaciones Marinas (CSIC), Vigo 36208, Spain \\ ${ }^{2}$ Departamento de Biología Animal, Instituto Cavanilles de Biodiversidad y Biología Evolutiva, Universitat de València, \\ 46701 Burjassot, Valencia, Spain \\ ${ }^{3}$ Centro Tecnológico del Mar, Fundación Cetmar, Vigo 36208, Spain
}

\begin{abstract}
In the mesozooplanktonic community of the coastal upwelling system of the Ría de Vigo (NW Spain), the euphausiid Nyctiphanes couchii has been identified for the first time in temperate waters of the NE Atlantic as the intermediate host for cystacanths of Rhadinorhynchus sp. Parasites were identified using morphological characters described in 20 cystacanths. The hooks of the proboscis were arranged in 14 rows of 26 hooks each, while the hooks of the basal circle were only slightly erected and were longer than remaining spines. A maximum-likelihood estimation (ML) tree inferred from the 18S rRNA data set of Palaeacantocephala revealed that our specimens belong to a highly supported clade with Rhadinorhynchus sp., Pararhadinorhynchus sp. and Transvena annulospinosa. Nonetheless, our morphological and phylogenetic analyses suggested that the status of Rhadinorhynchus pristis should be re-examined. The prevalences of parasites were $0.0019 \%$ and $0.0001 \%$ for frontal and coastal summer communities, and $0.0068 \%$ and $0.0008 \%$ for coastal and oceanic autumn communities, respectively. The presence of these cystacanths in different mesozooplankton communities throughout the study suggests that the recruitment of parasites may be affected by the oceanography.
\end{abstract}

KEY WORDS: Cystacanth · Euphausiid $\cdot$ Nyctiphanes couchii $\cdot$ Rhadinorhynchus sp. $\cdot$ Zooplankton · Acanthocephalan $\cdot$ NE Atlantic

Resale or republication not permitted without written consent of the publisher

\section{INTRODUCTION}

Acanthocephalans, or thorny-headed worms, are in adult stages obligate endoparasites of the intestine of vertebrates (Nickol 1985, Marcogliese 1995, Garey et al. 1996, Nickol et al. 2002, Taraschewski 2005). The cystacanths of this genus are the infective stage. Morphologically, they are similar to the mature worms, but differ in the size of the trunk and the degree of development of the sexual organs (Hoberg et al. 1993, Zdzitowiecki 1991).

Among zooplankton communities, euphausiids play an important role as intermediate hosts in the pelagic realm (Marcogliese 1995). They are able to attain massive biomasses that form vast and dense swarms occupying one of the lowest trophic levels. Moreover, they can be used by different parasites to reach their definitive host (Mauchline 1980, 1984, Marcogliese 2002). Nyctiphanes couchii (Bell, 1853) is the main euphausiid in the European continental shelf and one of its areas of higher concentrations is situated near the Spanish coast (Roura et al. 2013). This species is also one of the main prey items of different fish species, which in turn are involved in the diet of potential definitive hosts (Pascual et al. 1996, Marcogliese 2002). Some reports (e.g. Sars 1885, Shi- 
mazu 1975, Lindley 1977, Tsimbalyuk 1980, GómezGutiérrez et al. 2010, Gregori et al. 2012) recognised some species of cystacanths infecting the thoracic organs of adults of several species of krill.

Moreover, it is well-known that within the Palaeacanthocephala, a few members of Rhadinorhynchus (Lühe, 1911) have experienced considerable speciation in aquatic environments (Taraschewski 2005), where they are able to infect important commercial fishes such as Scombridae (Rego et al. 1985, Rego 1987), Xiphidae (Hogans et al. 1983), Belonidae, Carangidae, and Bramidae in the Atlantic Ocean (www.nhm.ac.uk/research-curation/research/projects/ host-parasites/database/index.jsp).

As far as we know, no previous data on the presence of Rhadinorhynchus sp. (Rudolphi, 1802) in euphausiids from the NE Atlantic are available. With the exception of Rodrigues et al. (1975) and Rego et al. (1985), Rhadinorhynchus has not been reported in this area. In addition, these authors found adults of this genus infecting scombrid fishes.

Despite parasites having a great ecological and economic significance in NE Atlantic waters, their recruitment to the zooplankton level is poorly understood. Therefore, the aims of this study were to (1) report the role of euphausiids in the life cycle of Rhadinorhynchus sp. in NW Iberian Peninsula waters; (2) provide data about parasite morphology, genetic and demographic infection values; and (3) discuss the controversy with the genetic identification of $R$. prisits (Rudolphi, 1802). equipped with a current meter, which enabled calculation of the volume of water filtered during the haul, thus permitting an estimation of zooplankton abundance $\left(\right.$ no. $\left.\mathrm{m}^{-3}\right)$. The sample was filtered using a $500 \mu \mathrm{m}$ sieve and fixed on board with $100 \%$ ethanol. Samples were later transferred to $70 \%$ ethanol in the laboratory and stored at $-20^{\circ} \mathrm{C}$.

\section{Zooplankton estimation}

The abundance of the different zooplankton taxa was estimated after counting a sub-sample using a Folsom splitter (Omori \& Ikeda 1984). Organisms were identified to the lowest possible taxonomic level. Species diversity was calculated using the Shannon-Weaver and Evenness indices (Omori \& Ikeda 1984, Guisande et al. 2006). The number of euphausiids was estimated using the method of calculating precise replica (Andrew \& Mapstone 1987).

\section{Collection and processing of cystacanths}

All zooplankton components of the samples were examined for acanthocephalans using a stereomicroscope (20x). Parasites were removed from the host using dissection material under the stereomicroscope. Cystacanths were identified by examining the body and proboscis according to Petrochenko (1956, 1958), Cable \& Linderoth (1963), Yamaguti (1963), Zdzitowiecki (1989), and Arai (1989). The number

\section{MATERIALS AND METHODS}

\section{Biological sampling}

The zooplankton samples were caught in the Ria de Vigo in Galician waters, NW Iberian Peninsula, onboard the RV 'Mytilus' (Fig. 1). Ten surveys were undertaken in the summer $(2,4,9$ and 11 July) and autumn (26 September, and 1, 3, 9, 10 and 14 October) of 2008. Samples were collected by double oblique towing, using a $750 \mathrm{~mm}$ diameter bongo net equipped with $375 \mu \mathrm{m}$ mesh. At a ship's speed of 2 knots, the bongo net was first lowered and stabilized near the bottom for a period of $15 \mathrm{~min}$, then hauled to the surface at $0.5 \mathrm{~m} \mathrm{~s}^{-1}$. The Bongo was

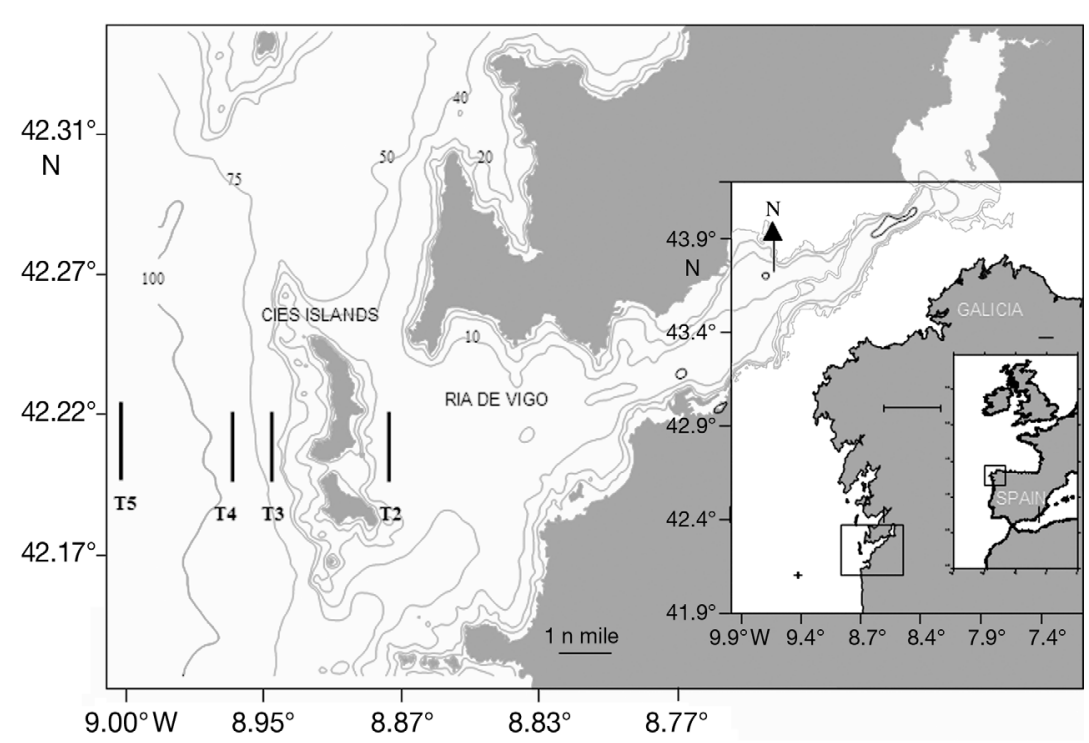

Fig. 1. Sampling area off the Ría de Vigo in Galician waters, NE Atlantic. T2-5: transects 2-5 
and distribution patterns of the proboscis armature and variations in the spination of the anterior part of body are considered the most important determining features (Miller \& Dunagan 1985). Morphological study was carried out to determine the cystcanth species. As the cystacanths normally presented the proboscis, neck, and part of the anterior trunk introverted, we dissected them to evert these structures. The body was cleared using the protocol described by Gregori et al. (2012) because this method does not damage DNA. The caudal part of the body was used for DNA extraction.

Scanning electron microscopy preparations in a Philips XL 30 were used to clarify the morphological examination. Infection parameters were estimated following Bush et al. (1997) and Rózsa et al. (2000). Sterne's exact $95 \%$ confidence interval (CI) was calculated for prevalence (Reiczigel 2003).

\section{Genomic DNA extraction and PCR amplification}

Genomic DNA was isolated using the Qiagen DNeasy ${ }^{\mathrm{TM}}$ Tissue Kit according to the manufacturer's instructions. DNA quality and quantity was checked in a NanoDrop ${ }^{\circledR}$ ND-1000 spectrophotometer and in $1 \%$ agarose gel. The primers 18SU467F (5'-ATC CAA GGA AGG CAG CAG GC-3') and 18SL1310R (5'-CTC CAC CAA CTA AGA ACG GC-3') (Suzuki et al. 2008) were employed to amplify approximately $900 \mathrm{bp}$ of the small subunit (18S) ribosomal RNA gene. PCRs were performed in a total volume of $25 \mu \mathrm{l}$ containing $1 \mu \mathrm{l}$ of genomic DNA (150-200 ng), PCR buffer at $1 \times$ concentration, $1.5 \mathrm{mM} \mathrm{MgCl}, 0.2 \mathrm{mM}$ nucleotides (Roche Applied Science), $0.3 \mu \mathrm{M}$ primers and 0.625 U Taq DNA polymerase (Roche Applied Science). The cycling protocol for 18S rRNA gene was 2 min at $94^{\circ} \mathrm{C}, 35$ cycles with $30 \mathrm{~s}$ at $94^{\circ} \mathrm{C}, 1 \mathrm{~min}$ at $55^{\circ} \mathrm{C}$ and 2 min at $72^{\circ} \mathrm{C}$, followed by 7 min at $72^{\circ} \mathrm{C}$. All PCRs were carried out in a TGradient thermocycler (Biometra) and a negative control (no DNA) was included for each set of PCRs.

\section{DNA sequencing and phylogenetic analysis}

Positive PCR products were cleaned for sequencing using ExoSAP-IT ${ }^{\odot}$ (USB Corporation). Sequences were subjected to BLASTn analyses against available sequences from GenBank, through web servers of the National Center for Biotechnology Information (USA). All 18S rRNA sequences present in GenBank of the Class Palaeacanthocephala were
Table 1. Species and GenBank accession numbers of taxa used for $18 \mathrm{~S}$ rDNA analyses

\begin{tabular}{|c|c|}
\hline Taxon & GenBank accession no. \\
\hline Southwellina hispida & JX014228, EU267809 \\
\hline Arhythmorhynchus brevis & AF064812 \\
\hline Pseudocorynosoma constrictum & EU267800 \\
\hline Ibirhynchus dimorpha & CQ981436 \\
\hline Hexaglandula corynosoma & EU267808 \\
\hline Pseudocorynosoma anatarium & EU267801 \\
\hline Polymorphus minutus & EU267806 \\
\hline Profilicollis botulus & EU267805 \\
\hline Polymorphus sp. & AF064815 \\
\hline Polymorphus altmani & AF001838 \\
\hline Andracantha gravida & EU267802 \\
\hline Corynosoma enhydri & AF001837 \\
\hline Corynosoma magdaleni & EU267803 \\
\hline Corynosoma strumosum & EU267804 \\
\hline Bolbosoma vasculosum & JX014225 \\
\hline Bolbosoma balaenae & JQ040306 \\
\hline Plagiorhynchus cylindraceus & AF001839 \\
\hline Centrorhynchus conspectus & U41399 \\
\hline Centrorhynchus sp. & AY830155 \\
\hline Centrorhynchus microcephalus & AF064813 \\
\hline Serrasentis sagittifer & JX014227 \\
\hline Gorgorhynchoides bullocki & AY830154 \\
\hline Transvena annulospinosa & AY830153 \\
\hline Pararhadinorhynchus sp. & HM545903 \\
\hline Rhadinorhynchus pristis & JQ061133 \\
\hline Rhadinorhynchus sp. & AY062433 \\
\hline Acanthocephalus dirus & AY830151 \\
\hline Acanthocephalus lucii & AY830152 \\
\hline Filisoma bucerium & AF064814 \\
\hline Filisomaa rizalinum & JX014229 \\
\hline Echinorhynchida sp. & EU732662 \\
\hline Acanthocephaloides propinquus & AY830149 \\
\hline Echinorhynchus gadi & $\begin{array}{c}\text { AY218123, U88335, } \\
\text { JX014222 }\end{array}$ \\
\hline Rhadinorhynchus pristis & JX014226 \\
\hline Rhadinorhynchus lintoni & JX14224 \\
\hline Pomphorhynchus laevis & $\begin{array}{c}\text { JX014223, AY218124, } \\
\text { AY423346 }\end{array}$ \\
\hline Pomphorhynchus tereticollis & AY423347 \\
\hline Dentitruncus truttae & JX460865 \\
\hline Illiosentis sp. & AY830158 \\
\hline Pseudoleptorhynchoides lamothei & i $\quad$ EU090950 \\
\hline Koronacantha pectinaria & AF092433, AY830157 \\
\hline Leptorhynchoides thecatus & AF001840 \\
\hline Pomphorhynchus bulbocoli & AF001841 \\
\hline Outgroup: Rotaria rotatoria & AY218121 \\
\hline Plationus patulus & DQ297712 \\
\hline
\end{tabular}

downloaded for phylogenetic analyses $(\mathrm{n}=49)$. Additionally, 2 rotiferan sequences were downloaded as an outgroup, due to their close relationship with the acanthocephalans (García-Varela et al. 2000). Table 1 shows the species used for phylogenetic analyses and their accession numbers. These $18 \mathrm{~S}$ rRNA sequences were aligned using MAFFT v5.7 (Katoh et al. 2002) with default settings. GBlocks 
(Castresana 2000) were then used to identify and remove highly divergent regions and poorly aligned positions. Afterwards, a substitution model was selected under Akaike's information criterion (Akaike 1974) as implemented in jModeltest (Posada 2008). The GTR+I+G (Tavaré 1986) model was chosen to infer the evolutionary history using the maximum likelihood (ML) method. The analysis involved 51 nucleotide sequences with a total of 582 conserved sites in the final data set. Bootstrap probabilities with 1000 replications were calculated to assess reliability on each node of the ML tree. Evolutionary analyses were conducted in MEGA5 (Tamura et al. 2011).

\section{RESULTS}

A total of 20 cystacanths infecting Nyctiphanes couchii were found in different samples. Their prevalences $(95 \% \mathrm{CI})$ are presented in Table 2 . Estimations of accompanying zooplankton taxa and abundance, Shannon-Weaver index and species evenness where larvae were found are recorded in Table 3. Complete information about mesozooplankton composition in each community is available in Roura et al. (2013). Cystacanths were removed from the thoracic organs (Fig. 2A) of $N$. couchii adults. The body of the cystacanths was cylindrical and in most samples their neck and proboscis were invaginated. A detailed description is given for male and female specimens pooled because sex could not always be determined. Morphometric measurements are given as means $\pm \mathrm{SD}$ [range], with sample size in parentheses.

\section{Description}

Trunk long, uniformly cylindrical, $10.5 \pm 3.9 \mathrm{~mm}$ [5.3-19.5 mm] long $(\mathrm{n}=18) \times 0.55 \pm 0.37 \mathrm{~mm}$ [0.33-1.87 mm] wide $(\mathrm{n}=15)$, spinose anteriorly. Trunk spines in 2 fields separated by unarmed zone (Fig. 2B,C). Anterior trunk spines adjacent to neck, arranged in 2 or 3 circles, the 3 rd ventrally not complete, posterior field restricted to ventral area (Fig. 2B), with ca. 10-14 rows of spines, reaching 23 $\pm 3.5 \%[15-28 \%]$ of trunk length $(\mathrm{n}=15)$. Trunk spines $82 \pm 20 \mu \mathrm{m}[53-118 \mu \mathrm{m}]$ long $(\mathrm{n}=163$ from 12 specimens), slightly longer in the posterior region, embedded in cuticular sheath. Neck cylindrical, 0.22 $\pm 0.10 \mathrm{~mm}[0.13-0.39 \mathrm{~mm}]$ long $\times 0.18 \pm 0.06 \mathrm{~mm}$ [0.12-0.23 mm] wide $(\mathrm{n}=5)$. Proboscis slender, cylindrical, $2.26 \pm 0.43 \mathrm{~mm}[1.55-3.32 \mathrm{~mm}]$ long $\times 0.20 \pm$ $0.06 \mathrm{~mm}$ [0.11-0.33 mm] wide $(\mathrm{n}=16)$ (Fig. 2D). Hooks arranged in 14 (rarely 13) rows of 26 hooks (rarely 23-25) each $(n=16)$. Hooks of basal circle only slightly erected and longer than remaining spines (Fig. 2D). Range of hook (H) length (in $\mu \mathrm{m}$ ) at base as follows ( $\mathrm{n}=10$ specimens except in H24-26, for which $\mathrm{n}=6$ ): H1: 93-131; H2: 62-99; H3: 79-98; H4: 67-110; H5: 74-110; H6: 69-111; H7: 78-120; H8: 87-114; H9: 88-124; H10: 94-128; H11: 90-123; H12: 91-122; H13: 94-118; H14: 86-110; H15: 77-111; H16: 85-123; H17: 96-122; H18: 87-113; H19: 92-116; H20: 85-114; H21: 77-122; H22: 85-111; H23: 68-108; H24: 85-99; H25: 68-98; H26: 66-88. Proboscis receptacle considerably longer than proboscis, $3.8 \pm 0.5 \mathrm{~mm}[2.9-4.7 \mathrm{~mm}]$ long $(\mathrm{n}=16) \times$ $0.18 \pm 0.06[0.22-0.28] \mathrm{mm}$ wide $(\mathrm{n}=4)$. Lemnisci not extending past receptacle, generally hidden behind

Table 2. Nyctiphanes couchii population divided into 3 different stages in each community during 2008, where cystacanths were found from Ría de Vigo, Galicia, NW Atlantic, Spain, in different samplings: summer frontal (SF), summer coastal (SC), autumn ocean (AO) and autumn coastal (AC). T5B: transect 5 at bottom; T2B: transect 2 at bottom; T3B: transect 3 at bottom. N: number of individuals estimated; $\mathrm{Ab}$ : abundance $\left(\text { no. }^{-3}\right)_{i}$ Population \% [CI]: prevalence in the population [95\% confidence interval]; Adults $\%[\mathrm{CI}]:$ prevalence in adults [95\% confidence interval]

\begin{tabular}{|c|c|c|c|c|c|c|c|c|c|c|c|c|}
\hline \multirow{3}{*}{ Community } & \multirow{2}{*}{\multicolumn{2}{|c|}{$-\mathrm{SF}-\overline{\mathrm{T} 5 \mathrm{~B}, 2 \mathrm{Jul}}$}} & \multirow{2}{*}{\multicolumn{2}{|c|}{$\begin{array}{l}\mathrm{SC} \\
\mathrm{T} 2 \mathrm{~B}, 2 \mathrm{Jul}\end{array}$}} & \multirow{2}{*}{\multicolumn{2}{|c|}{ T5B, 26 Sep }} & \multirow{2}{*}{\multicolumn{2}{|c|}{$\begin{array}{c}\mathrm{AO} \\
\mathrm{T} 5 \mathrm{~B}, 1 \mathrm{Oct}\end{array}$}} & \multirow{2}{*}{\multicolumn{2}{|c|}{ T5B, 9 Oct }} & \multirow{2}{*}{\multicolumn{2}{|c|}{$\begin{array}{l}\mathrm{AC} \\
\mathrm{T} 3 \mathrm{~B}, 10 \mathrm{Oct}\end{array}$}} \\
\hline & & & & & & & & & & & & \\
\hline & $\mathrm{N}$ & $\mathrm{Ab}$ & $\mathrm{N}$ & $\mathrm{Ab}$ & $\mathrm{N}$ & $\mathrm{Ab}$ & $\mathrm{N}$ & $\mathrm{Ab}$ & $\mathrm{N}$ & $\mathrm{Ab}$ & $\mathrm{N}$ & $\mathrm{Ab}$ \\
\hline Calyptopis & 36433 & 88.78 & 1423426 & 2097.96 & 2496 & 11.30 & 4060 & 25.52 & 475 & 2.13 & 165120 & 436.46 \\
\hline Furcilia & 49388 & 120.35 & 37415 & 55.15 & 4512 & 20.44 & 480 & 3.02 & 175 & 0.78 & 92160 & 243.61 \\
\hline Adults & 69955 & 170.47 & 328 & 0.48 & 3600 & 16.31 & 2620 & 16.47 & 2175 & 9.76 & 1920 & 5.07 \\
\hline Population & 155775 & 380.60 & 1461169 & 2154.59 & 10608 & 48.05 & 7160 & 45.01 & 2825 & 13.67 & 259200 & 685.14 \\
\hline No. infected & \multicolumn{2}{|c|}{3} & \multicolumn{2}{|c|}{1} & \multicolumn{2}{|c|}{12} & \multicolumn{2}{|c|}{1} & \multicolumn{2}{|c|}{1} & \multicolumn{2}{|c|}{2} \\
\hline Population \% [CI] & \multicolumn{2}{|c|}{$\begin{array}{c}0.0019 \\
{[0.0004-0.002]}\end{array}$} & \multicolumn{2}{|c|}{$\begin{array}{c}0.0001 \\
{[0.0000-0.00013]}\end{array}$} & \multicolumn{6}{|c|}{$\begin{array}{c}0.068 \\
{[0.0372-0.0445]}\end{array}$} & \multicolumn{2}{|c|}{$\begin{array}{c}0.0008 \\
{[0.0001-0.0008]}\end{array}$} \\
\hline Adults \% [CI] & \multicolumn{2}{|c|}{$\begin{array}{c}0.0043 \\
{[0.0125-0.0009]}\end{array}$} & \multicolumn{2}{|c|}{$\begin{array}{c}0.3047 \\
{[0.0000-0.0169]}\end{array}$} & \multicolumn{6}{|c|}{$\begin{array}{c}0.0014 \\
{[0.0912-0.2797]}\end{array}$} & \multicolumn{2}{|c|}{$\begin{array}{c}0.1042 \\
{[0.0126-0.3758]}\end{array}$} \\
\hline
\end{tabular}


Table 3. Mesozooplankton taxa collected in each community during 2008, where cystacanths were found from Ría de Vigo, Galicia, NW Atlantic, Spain, in different samplings: summer frontal (SF), summer coastal (SC), autumn ocean (AO) and autumn coastal (AC). T5B: transect number 5 at bottom; T2B: transect 2 at bottom; T3B: transect 3 at bottom. N: number of individuals estimated; Ab: abundance $\left(\right.$ no. $\mathrm{m}^{-3}$ ). Volumes filtered per transect were $410.37,678.48,220.78,159.07,222.92$, and $3783.2 \mathrm{~m}^{3}$, respectively. -: taxon not found

\begin{tabular}{|c|c|c|c|c|c|c|c|c|c|c|c|c|}
\hline \multirow[t]{2}{*}{ Community } & \multicolumn{2}{|c|}{ T5B, 2 Jul } & \multicolumn{2}{|c|}{$\begin{array}{c}\mathrm{SC} \\
\mathrm{T} 2 \mathrm{~B}, 2 \mathrm{Jul}\end{array}$} & \multicolumn{2}{|c|}{ T5B, 26 Sep } & \multicolumn{2}{|c|}{$\begin{array}{c}\mathrm{AO} \\
\mathrm{T} 5 \mathrm{~B}, 1 \mathrm{Oct}\end{array}$} & \multicolumn{2}{|c|}{ T5B, 9 Oct } & \multicolumn{2}{|c|}{$\begin{array}{c}\mathrm{T} 3 \mathrm{~B}, 10 \mathrm{Oct} \\
\end{array}$} \\
\hline & $\mathrm{N}$ & $\mathrm{Ab}$ & $\mathrm{N}$ & $\mathrm{Ab}$ & $\mathrm{N}$ & $\mathrm{Ab}$ & $\mathrm{N}$ & $\mathrm{Ab}$ & $\mathrm{N}$ & $\mathrm{Ab}$ & $\mathrm{N}$ & $\mathrm{Ab}$ \\
\hline \multicolumn{13}{|l|}{ MEROPLANKTON } \\
\hline \multicolumn{13}{|l|}{ Cephalopoda } \\
\hline$\overline{\text { Loliginidae }}$ & - & - & 8 & 0.01 & 2 & 0.01 & - & & 1 & 0.004 & 4 & 0.01 \\
\hline Octopus vulgaris & - & - & 1 & 0.001 & 1 & 0.005 & 3 & 0.01 & 2 & 0.01 & 13 & 0.03 \\
\hline Sepiolidae & 7 & 0.08 & 1 & 0.001 & - & - & - & - & - & - & 3 & 0.01 \\
\hline \multicolumn{13}{|l|}{ Cirripedia } \\
\hline$\overline{\text { Cirripedia larvae }}$ & - & - & 4267 & 6.29 & - & - & - & - & - & - & 170880 & 451.69 \\
\hline \multicolumn{13}{|l|}{ Echinodermata } \\
\hline$\overline{\text { Echinoidea larvae }}$ & - & - & 1422 & 2.10 & - & - & - & - & - & - & 3840 & 10.15 \\
\hline Ophiuroidea larvae & 512 & 1.28 & 28444 & 41.92 & - & - & - & - & - & - & 353280 & 933.82 \\
\hline \multicolumn{13}{|l|}{ Fish } \\
\hline$\overline{\text { Fish eggs }}$ & - & - & - & - & - & - & - & - & - & - & 1920 & 5.07 \\
\hline Fish larvae & 512 & 1.25 & 1046 & 1.54 & 29 & 0.13 & 2 & 0.01 & 6 & 0.03 & 1969 & 5.20 \\
\hline \multicolumn{13}{|l|}{ Gastropoda } \\
\hline$\overline{\text { Gastropoda larvae }}$ & - & - & 12800 & 18.87 & - & - & - & - & 25 & 0.11 & 24960 & 65.98 \\
\hline \multicolumn{13}{|l|}{ Isopoda } \\
\hline$\overline{\text { Aegidae }}$ & - & - & 50 & 0.07 & - & - & - & - & - & - & 24 & 0.06 \\
\hline \multicolumn{13}{|l|}{ Decapoda } \\
\hline$\overline{\text { Alpheidae zoeae }}$ & - & - & 1422 & 2.10 & - & - & - & - & 25 & 0.11 & - & - \\
\hline Brachyura juvenile & - & - & 7 & 0.01 & 2 & 0.01 & 1 & 0.01 & - & - & - & - \\
\hline Brachyura megalopa & - & - & - & & - & - & - & - & - & - & 1920 & 5.07 \\
\hline Brachyura zoeae & 2463 & 6.00 & 45511 & 67.08 & - & - & 40 & 0.25 & 25 & 0.11 & 24960 & 65.98 \\
\hline Crangonidae zoeae & - & - & 1422 & 2.10 & - & - & - & & - & - & - & \\
\hline Paguridae megalopa & 480 & 1.17 & & & - & - & - & - & - & - & - & \\
\hline Paguridae zoeae & - & - & 4267 & 6.29 & - & - & 20 & 0.13 & - & - & 9600 & 25.38 \\
\hline Palaemonidae zoeae & - & - & 1422 & 2.10 & - & - & - & - & - & - & - & \\
\hline Pisidia longicornis megalope & $\mathrm{a}-$ & - & - & & - & - & - & - & - & - & 1920 & 5.07 \\
\hline Pisidia longicornis zoeae & - & - & 15644 & 23.06 & - & - & - & - & - & - & 17280 & 45.68 \\
\hline Porcellana platycheles zoeae & e - & - & 5689 & 8.38 & - & - & - & - & - & - & 15360 & 40.60 \\
\hline Processidae zoeae & - & - & 533 & 0.92 & - & - & - & - & - & - & 600 & 11.41 \\
\hline Jaxea nocturna & - & - & 1422 & 2.10 & - & - & - & - & - & - & - & - \\
\hline \multicolumn{13}{|l|}{ Amphipoda } \\
\hline$\overline{\text { Caprellidea }}$ & - & - & 6 & 0.009 & - & - & 4 & 0.02 & 1 & 0.004 & - & - \\
\hline Gammaridea & 85 & 0.201 & 65 & 0.10 & 6 & 0.03 & 2 & 0.01 & 24 & 0.11 & 176 & 0.46 \\
\hline \multicolumn{13}{|l|}{ Stomatopoda } \\
\hline$\overline{\text { Meiosquilla desmaresti }}$ & 480 & 1.17 & - & - & - & - & - & - & - & - & - & - \\
\hline Polychaeta & & & & & & & & & & & & \\
\hline$\overline{\text { Polychaeta larvae }}$ & 5 & 0.01 & 49 & 0.07 & 35 & 0.16 & 6 & 0.04 & 8 & 0.04 & 21 & 0.06 \\
\hline HOLOPLANKTON & & & & & & & & & & & & \\
\hline Appendicularia & - & - & 7111 & 10.48 & - & - & - & - & - & - & 71040 & 187.78 \\
\hline Amphipoda & & & & & & & & & & & & \\
\hline$\overline{\text { Hyperiidea }}$ & - & - & 1 & 0.001 & 5 & 0.02 & 9 & 0.06 & 17 & 0.08 & 13 & 0.03 \\
\hline Chaetognatha & - & - & 11942 & 17.60 & 1861 & 8.43 & 1220 & 7.67 & 775 & 3.48 & 96000 & 253.76 \\
\hline Cnidaria & - & - & - & - & - & - & - & - & - & - & 5760 & 15.22 \\
\hline Cladocera & & & & & & & & & & & & \\
\hline$\overline{\text { Evadne nordmanni }}$ & - & - & 14222 & 20.96 & - & - & - & - & - & - & 36480 & 96.43 \\
\hline Podon intermedius & - & - & 36978 & 54.50 & - & - & - & - & - & - & 55680 & 147.18 \\
\hline Hydrozoa & & & & & & & & & & & & \\
\hline Siphonophora & 1536 & 3.74 & 5317 & 7.84 & 74 & 0.33 & - & - & 300 & 1.35 & 1920 & 5.07 \\
\hline
\end{tabular}


Table 3 (continued)

\begin{tabular}{|c|c|c|c|c|c|c|c|c|c|c|c|c|}
\hline \multirow[t]{3}{*}{ Community } & \multirow{2}{*}{\multicolumn{2}{|c|}{$\underset{\mathrm{T} 5 \mathrm{~B}, 2 \mathrm{Jul}}{ }$}} & \multirow{2}{*}{\multicolumn{2}{|c|}{$-\mathrm{SC}=$}} & \multirow{2}{*}{\multicolumn{4}{|c|}{$\begin{array}{ll} & \mathrm{AO} \\
\mathrm{T} 5 \mathrm{~B}, 26 \mathrm{Sep} & \mathrm{T} 5 \mathrm{~B}, 1 \mathrm{Oct}\end{array}$}} & \multirow{2}{*}{\multicolumn{2}{|c|}{ T5B, 9 Oct }} & \multirow{2}{*}{\multicolumn{2}{|c|}{$-\mathrm{AC}=\overline{\mathrm{T} 3 \mathrm{~B}, 10 \mathrm{Oct}}$}} \\
\hline & & & & & & & & & & & & \\
\hline & $\mathrm{N}$ & $\mathrm{Ab}$ & $\mathrm{N}$ & $\mathrm{Ab}$ & $\mathrm{N}$ & $\mathrm{Ab}$ & $\mathrm{N}$ & $\mathrm{Ab}$ & $\mathrm{N}$ & $\mathrm{Ab}$ & $\mathrm{N}$ & $\mathrm{Ab}$ \\
\hline \multicolumn{13}{|l|}{ Euphausiacea } \\
\hline $\begin{array}{l}\text { Nyctiphanes couchii } \\
\text { calyptopis }\end{array}$ & 36433 & 88.78 & 1423426 & 2097.96 & 2496 & 11.30 & 4060 & 25.52 & 475 & 2.13 & 165120 & 436.46 \\
\hline N. couchii furcilia & 49388 & 120.35 & 37415 & 55.15 & 4512 & 20.44 & 480 & 3.02 & 175 & 0.78 & 92160 & 243.61 \\
\hline N. couchii adult & 69955 & 170.47 & 328 & 0.48 & 3600 & 16.31 & 2620 & 16.47 & 2175 & 9.76 & 1920 & 5.08 \\
\hline \multicolumn{13}{|l|}{ Copepoda } \\
\hline$\overline{\text { Acartia clausi }}$ & 7421 & 18.08 & 93867 & 138.35 & 1263 & 5.721 & 260 & 1.63 & 3350 & 15.03 & 99840 & 263.91 \\
\hline Candacia armata & - & - & 1422 & 2.10 & - & - & - & - & - & - & - & - \\
\hline Calanoides carinatus & 72259 & 176.08 & 2844 & 4.19 & 1600 & 7.25 & 20 & 0.13 & 575 & 2.58 & 7680 & 20.30 \\
\hline Calanus helgolandicus & 35156 & 85.67 & 1422 & 2.10 & 1853 & 8.39 & 100 & 0.63 & 475 & 2.13 & - & - \\
\hline Centropages chierchiae & 3966 & 9.67 & 14222 & 20.96 & - & - & 20 & 0.13 & 75 & 0.34 & 1920 & 5.07 \\
\hline Centropages typicus & - & - & - & - & - & - & - & - & - & - & 1920 & 5.07 \\
\hline Clausocalanus spp. & - & - & - & - & - & - & 100 & 0.63 & 100 & 0.45 & 3840 & 10.15 \\
\hline Diaixis pygmaea & - & - & 1422 & 2.10 & - & - & - & - & 25 & 0.11 & - & - \\
\hline Isias clavipes & - & - & 1422 & 2.10 & - & - & - & - & - & - & 1920 & 5.07 \\
\hline Mesocalanus tenuicornis & - & - & - & - & 253 & 1.14 & - & - & - & - & - & - \\
\hline Metridia lucens & - & - & 1422 & 2.10 & 1432 & 6.48 & - & - & 100 & 0.45 & - & - \\
\hline Paracalanus parvus & - & - & - & - & 253 & 1.14 & - & - & 575 & 2.58 & 1920 & 5.07 \\
\hline Paraeuchaeta hebes & 8637 & 21.05 & - & - & 7326 & 33.18 & 220 & 1.38 & 850 & 3.81 & 1920 & 5.07 \\
\hline Paraeuchaeta sp. & 4797 & 11.69 & - & - & 20463 & 92.68 & 1700 & 10.69 & 2225 & 9.98 & 15360 & 40.60 \\
\hline Pseudocalanus elongatus & - & - & - & - & - & - & 20 & 0.13 & 25 & 0.11 & - & - \\
\hline Subeucalanus crassus & - & - & - & - & - & - & 20 & 0.13 & - & - & - & - \\
\hline Temora longicornis & - & - & 28444 & 41.92 & - & - & - & - & - & - & 9600 & 25.38 \\
\hline Cyclopoidea & & & & & & & & & & & & \\
\hline Oithona plumifera & - & - & - & - & - & - & - & - & 250 & 1.12 & 5760 & 15.22 \\
\hline Mysidacea & 1919 & 4.68 & - & - & 528 & 2.39 & 360 & 2.26 & 475 & 2.13 & 1920 & 5.07 \\
\hline Thaliacea & & & & & & & & & & & & \\
\hline$\overline{\text { Salpida }}$ & 21175 & 51.60 & 4267 & 6.29 & 3874 & 17.54 & 1780 & 11.19 & 1900 & 8.52 & 13440 & 35.53 \\
\hline Polychaeta & & & & & & & & & & & & \\
\hline 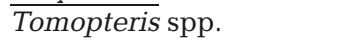 & - & - & - & - & - & - & - & - & 25 & 0.11 & - & - \\
\hline Total & 317185 & 773.93 & 1814417 & 2674.37 & 51466 & 233.11 & 13067 & 82.15 & 15084 & 67.66 & 1321863 & 3503.90 \\
\hline Shannon's Index $\left(H^{\prime}\right)$ & 1.55 & 0.507 & 2.12 & 1.49 & 2.31 & 2.37 & & & & & & \\
\hline Evenness index & 0.54 & 0.139 & 0.71 & 0.48 & 0.69 & 0.64 & & & & & & \\
\hline
\end{tabular}

it but apparently reaching half of proboscis receptacle $(\mathrm{n}=2)$. Terminal gonopore. A voucher specimen was deposited at the Natural History Museum of London, UK, with the accession number NHMUK 2013.4.2.1

Amplified sequences of 18S rRNA ranged from 814 to $819 \mathrm{bp}$. These sequences are available on GenBank under the accession numbers JQ061133-JQ061136. BLAST search showed close homology (98\%) with the 18S rRNA of Rhadinorhynchus sp. and Pararhadinorhynchus sp. (Johnston \& Edmonds, 1947). The ML tree inferred from the 18S rRNA data set of Palaeacantocephala revealed that our specimens belong to a highly supported clade (bootstrap values of 100), with Rhadinorhynchus sp., Pararhadinorhynchus sp. and Transvena annulospinosa (Pichelin \& Cribb 2001) (Fig. 3). Unexpectedly, sequences of Rhadinorhyn- chus pristis and $R$. lintoni (Cable \& Linderoth 1963), recently described by Verweyen et al. (2011), are nested in a highly supported group with Pomporhynchus (Monticelli, 1905) species, displaying homologies of $99 \%$ with those species. Comparing our data against the sequences of Verweyen et al. (2011) revealed homology of only $84 \%$. The ML tree showed the monophyly of Polymorphida and the paraphyly of Echinorhynchida, the 2 orders found within Palaeacantocephala. In fact, Rhadinorhynchidae was the most polyphyletic family among the Echinorhynchida.

\section{DISCUSSION}

Cystacanths found in Nyctiphanes couchii can be undoubtedly assigned to the genus Rhadinorhynchus 

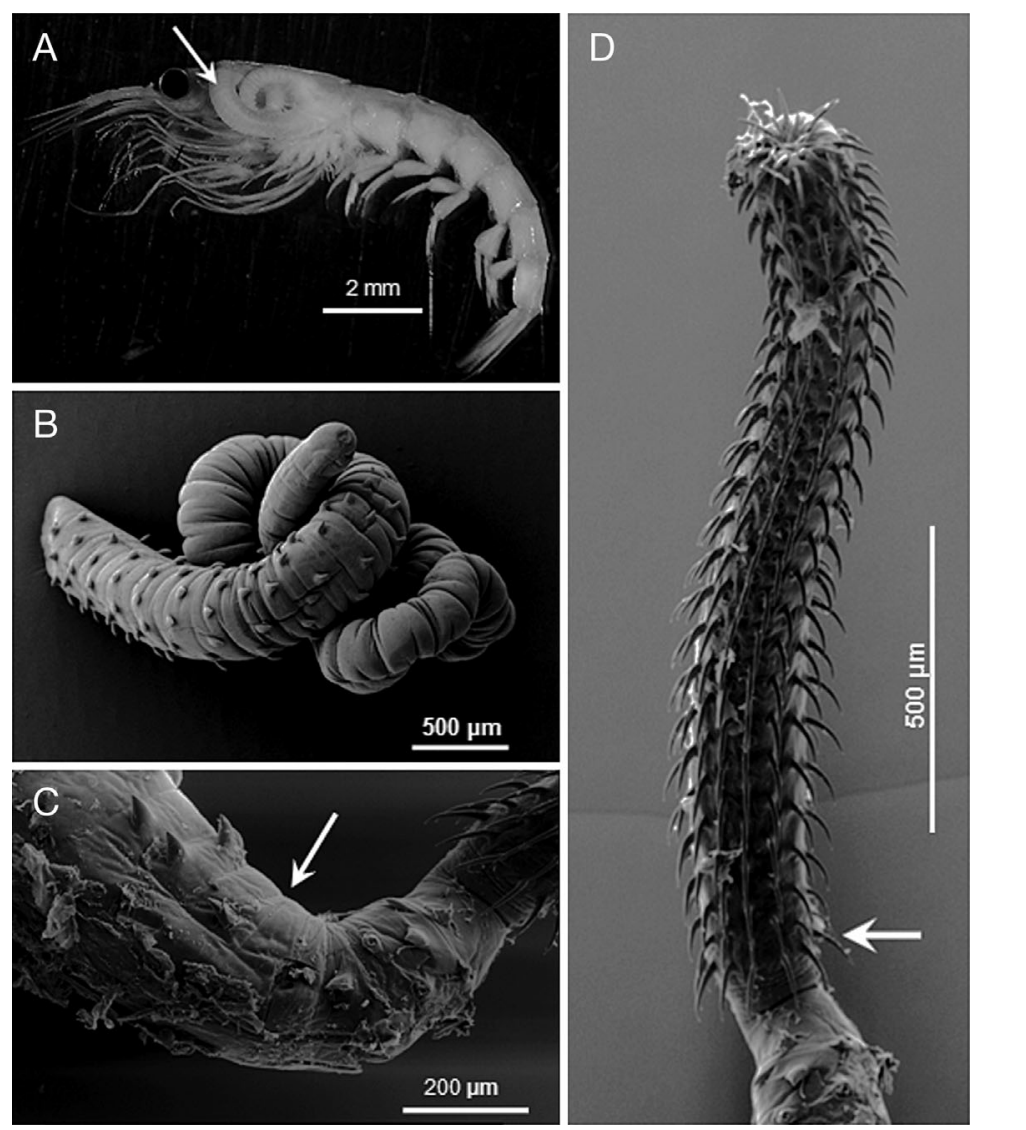

Fig. 2. Cystacanth of Rhadinorhynchus sp. from Nyctiphanes couchii. (A) Single infection with one cystacanth of Rhadinorhynchus sp. in the cephalothorax cavity (arrow). (B) The spines of the anterior part of the body, general view. (C) Two fields of spines separated by a space without spines (arrow). (D) Cystacanth's proboscis. Basal circle perpendicular hooks (arrow)

based on the patterns of trunk armature and proboscis morphology (see Petrochenko 1956, Golvan 1969, Amin et al. 2011). Pararhadinorhynchus and Transvena, which belong to the same clade as Rhadinorhynchus (Fig. 3), can be readily distinguished using their taxonomy because a key character for species of the genus Pararhadinorhynchus is the absence of trunk spines. The morphotype of species of Transvena is distinguished from other acanthocephalans because their trunk possesses a single ring of posteriorly pointing spines, at or near the junction between the neck and trunk (Pichelin \& Cribb 2001). In contrast, all our examined specimens possess extended fields of spines on the trunk.

According to the most recent key to species of Rhadinorhynchus (Amin et al. 2011), our specimens belong to a group of 20 species that combine 2 character states that are apparently stable: (1) trunk spines in 2 fields clearly separated by an unarmed zone; and (2) dorsal spines absent in the posterior field. Among these, only a single species, namely Rhadinorhynchus saltatrix (Troncy \& Vassiliades 1973), exhibits the combination of a proboscis armature with 14 rows of hooks and a field of posterior trunk spines with $>10$ spine rows (Amin et al. 2011). The description of $R$. saltatrix is well detailed but based only on a few specimens, 5 males and 8 females (Troncy \& Vassiliades 1973). Our specimens agree with the description made by Troncy \& Vassiliades (1973) except that the number of hooks per row that they report is 24 in $R$. saltatrix while 26 hooks per row are more frequently found in our specimens. However, it is important to note that our specimens closely resemble $R$. pristis and $R$. selkirki (Van Cleave, 1921), except that the number of spine rows in the posterior field of the trunk is smaller $(\leq 10)$ in these species (see Amin et al. 2011).

From the above results a key question is the range of variability of this spiny field, and the factors that may influence this trait. For instance, there is evidence of clear sexual differences of this trait in many species (see e.g. Petrochenko 1958, Cable \& Linderoth 1963, Troncy \& Vassiliades 1973). In contrast, the status of Rhadinorhynchus pristis is currently rather confused. Amin et al. (2011) list 7 species that, in their opinion, were erroneously identified as $R$. pristis, but they consider $R$. selkirki as a valid species. In contrast, Chandler (1934) and Petrochenko (1956) consider R. selkirki as synonym of $R$. pristis. To compound the problem, other available descriptions of $R$. pristis (e.g. Rego 1987, Arai 1989, Bunkley-Williams \& Williams 1996) were not included in the revision by Amin et al. (2011) and do not fulfil the diagnostic traits of this species sensu these authors.

According to the above morphological discussion, we should tentatively identify our specimens as Rhadinorhynchus saltatrix, pending a critical reexamination of $R$. pristis and related species.

Once the morphological identification was confirmed, genetic homology using 18S rRNA sequences allowed us to assign the cystacanths to the genus Rhadinorhynchus (Fig. 3). The 18S (SSU sequences) have been broadly used in different research as a taxonomic tool to clarify the taxonomy of acanthocephalans at the species level (Near et al. 1998, Gar- 


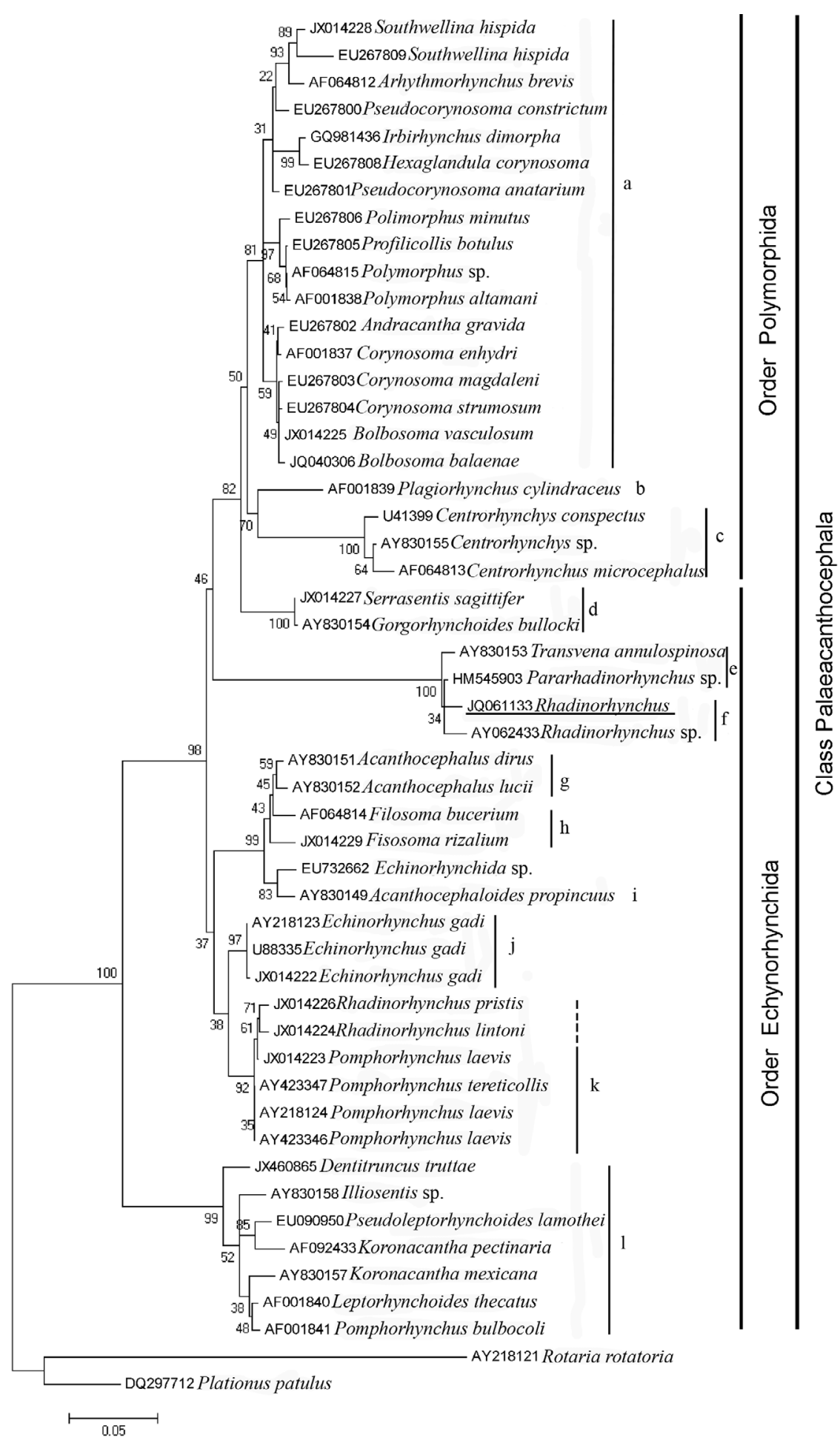

Fig. 3. Maximum likelihood consensus tree after 1000 bootstraps showing the phylogenetic relationships of the Palaeacanthocephala using 18S rRNA sequences and Rotifera as the outgroup. Abbreviations: a, Polymorphidae; b, Plagiorhynchidae; c, Centrorhynchidae; d, Rhadinorhynchidae; e, Transvenidae; f, Rhadinorhynchidae; g, Echinorhynchidae; h, Cavisomidae; i, Arhythmacanthidae; j, Echinorhynchidae; k, Pomphorhynchidae; l, Illiosentidae. Underlined species correspond with cystacanths found infecting Nyctiphanes couchii
cía-Varela et al. 2000, 2002, Herlyn et al. 2003, Near 2002, GarcíaVarela \& Nadler 2005, 2006, García-Varela \& González-Oliver 2008, Gregori et al. 2012). At the genetic level, our identification disagrees with the results obtained by Verweyen et al. (2011), whose sequences correspond with $R$. pristis and $R$. lintoni. Phylogenetic analyses showed almost the same topology, but the position of Rhadinorhynchus species is markedly different. Our sequences appeared in a well-supported clade with members of the family Transvenidae, Transvena annulospinosa and Rhadinorhynchus sp. as reported by García-Varela \& Nadler (2005) and García-Varela \& GonzálezOliver (2008). However, R. pristis and $R$. lintoni identified by Verweyen et al. (2011) appeared within the Pomporhynchus group. This contradiction is due to the absence of Pararhadinorhynchus sp. and Rhadinorhynchus sp. sequences in the Bayesian analysis carried out by Verweyen et al. (2011). The omission of these 2 sequences placed their $R$. pristis and $R$. lintoni far from Transvenna annulospinosa, which was a clade strongly supported with a bootstrap confidence of $100 \%$ (García-Varela \& Nadler 2005, García-Varela \& GonzálezOliver 2008). Our results suggest that genetic identification of Rhadinorhynchus by Verweyen et al. (2011) should be revised. Another explanation would be that Rhadinorhynchus is a polyphyletic genus, since Rhadinorhynchidae is a polyphyletic family as shown by morphological, molecular and cladistic studies (Herlyn et al. 2001, Monks 2001, García-Varela et al. 2002, García-Varela \& Nadler 2005, García-Varela \& González-Oliver 2008). It is clear that the paraphyly of Rhadinorhynchidae requires reexamination and reclassification and even the creation of new fami- 
lies. As suggested by Pichelin \& Cribb (2001) and García-Varela \& Nadler (2005), the group formed by Serrasentis sagittifer (Linton, 1889) and Gorgorhynchoides bullocki (Cable \& Mafarachisi, 1970), plus Golvanorhynchus (Noronha, Fabio \& Pinto, 1978), which form a sister group of Polymorphida, should be removed from the Rhadynorhynchidae. Our results support the transfer of Leptorhynchoides (Kostylev, 1924) and Pseudoleptorhynchoides (SalgadoMaldonado, 1976) (both Rhadinorhynchidae) to the family Illiosentidae, as suggested by García-Varela \& González-Oliver (2008). This way the Illiosentidae would be a monophyletic clade. In our work the order Polymorphida is monophyletic, in contrast with the works of García-Varela \& Nadler (2005), GarcíaVarela \& González-Oliver (2008) and Verweyen et al. (2011). This difference may result from the larger data set analysed in this work, 49 Palaeacantocephalan sequences, versus the 19, 20 and 36 used by García-Varela \& Nadler (2005), García-Varela \& González-Oliver (2008) and Verweyen et al. (2011), respectively. Apart from $R$. pristis and $R$. lintoni, our phylogenetic analysis highlights the possible misidentification of Pomporhynchus bulbocoli (Linkins in Van Cleave, 1919) as a member of the monophyletic clade Illiosentidae and Echinorhynchida sp. (Cobbold, 1879) (Fig. 3).

This is the first time that Rhadinorhynchus sp. has been found in the euphausiid Nyctiphanes couchii. Euphausiids are an essential and abundant nexus between the mesozooplankton and nekton, ingested by fishes, cephalopods (both acting as paratenic host) and birds (Deagle et al. 2007, Braley et al. 2010, Roura et al. 2012).

Reports of larval acanthocephalans acting as intermediate hosts in zooplankton are scarce. Among the Palaeacanthocephala some Echinorhynchus corrugatus have been found in Euphausia krohnii (Marcogliese 1995). Bolbosoma caenoforme has been found infecting Thysanoessa longipes and T. raschii (Shimazu 1975), whereas Bolbosoma sp. (Porta, 1908) has been detected in Thysanoessa sp. (Brandt, 1851) (Tsimbalyuk 1980). Lindley (1977) reported 3 larvae of Paleoacanthocephala infecting the euphausiid $T$. longicaudata. Recently, 3 larval stages of Polymorphida (Bolbosoma or Corynosoma) were reported within Nyctiphanes simplex on the northwestern coast of Mexico (Gómez-Gutiérrez et al. 2010). Finally, cystacanths of Bolbosoma balaenae were found in Nyctiphanes couchii in the NE Atlantic (Gregori et al. 2012). Therefore, only acanthocephalans the orders Echinorhynchida and Polymorphida have been found in euphausiids. This fact may be related to their final hosts, with Echinorhynchida infecting mainly teleost fishes and occasionally amphibians and reptiles, whereas Polymorphida infect mainly birds and marine mammals (Bush et al. 2001).

Rhadinorhynchus is a generalist at the definitive host level. However, at the zooplankton level, it demonstrated some specificity for krill. This specificity is reinforced because we did not find cystacanths of Rhadinorhynchus sp. in the larvae of $\mathrm{Nyc-}$ tiphanes couchii (calyptopis and furcilias with $0.8-2.20$ and $2.2-5.5 \mathrm{~mm}$ in total length, respectively). We also did not find them in copepod species or other taxa, probably due to the large size of these cystacanths $(\sim 11.41 \mathrm{~mm})$. We suspect that only adults of $N$. couchii could harbour them in their cephalothorax (12-17 mm length). Consequently, it seems that the smaller zooplankton organisms cannot harbour large cystacanths or act as intermediate hosts for these acanthocephalans. Moreover, the large size of the cystacanths we found probably acts to limit the intensity to 1 , because none of the examined $N$. couchii showed more than one cystacanth per individual, as described in Gómez-Gutiérrez et al. (2010) and Gregori et al. (2012).

The prevalence of Rhadinorhynchus sp. within the euphausiid population was very low (Table 2). This is usually considered a feature of the zooplankton trophic level because of the dilution effect of the pelagic realm where it becomes difficult to find a suitable intermediary host (Marcogliese 2002). Nevertheless, since most predators ingest large quantities of krill, euphausiids become significant intermediate or paratenic hosts that originate high infection rates and intensities in the final parasite's hosts (Marcogliese 1995, 2002). Despite the fact that low prevalence is a feature at the zooplankton level, information about it is very scarce. Nevertheless, our prevalences are similar to those reported by Shimazu (1975) with infection rates about $0.219 \%$ in Thysanoessa longipes and $13.33 \%$ in T. raschii. GómezGutiérrez et al. (2010) reported an average of prevalence about $3.1 \%$ in Nyctiphanes simplex and Gregori et al. (2012) reported a prevalence of $0.10 \%$ in $N$. couchii.

The ecological impact of cystacanths can be better understood if we consider the whole mesozooplanktonic community where the sample was taken. Roura et al. (2013) defined 6 characteristic mesozooplankton communities in the Ría de Vigo during the upwelling season following the bathymetric gradient, 3 in early summer and 3 in autumn, named as coastal, frontal and oceanic. These 6 communities 
changed from summer to autumn due to a shift from relaxation-downwelled to upwelled conditions coupled with life-cycle changes in the zooplankton. We found cystacanths in the coastal (SC) and frontal (SF) summer communities as well as coastal (AC) and oceanic (AO) autumn communities (Table 3). A total of 2079 and 185107 adults of Nyctiphanes couchii were counted in SC and SF, respectively, and therefore the number of potential infected adults would be 6 and 8 in these 2 communities. In autumn communities under upwelling conditions the number of adults of $N$. couchii was 3363 and 16741 individuals in AC and AO. Accordingly, 4 and 24 would be the inferred number of infected adults in each community, respectively. These results suggest that the recruitment of parasites may be affected by the oceanography (Pascual et al. 2007).

In conclusion, we would like to emphasize that this is the first record of Rhadinorhynchus sp. in Nyctiphanes couchii in coastal waters of the NW Iberian Peninsula, with $N$. couchii probably acting through predator-prey interactions as an intermediate host. The results of our morphological and phylogenetic study, along with the available epidemiological information on $R$. pristis infection in Scombridae and Xiphidae fishes from the nearby Portugal coast, the Madeira Islands and the North Atlantic, suggest that the cystacanths herein described probably belong to this species (Rodrigues et al. 1975, Vassiliades 1982, Hogans et al. 1983, Rego 1987, Costa et al. 2004). However, we strongly recommend that a thorough review of the species, as well as the family Rhadinorhynchidae, should be carried out.

Acknowledgements. The authors are grateful to many colleagues for assisting us with the collection and sorting of zooplankton, as well as the crew of the RV 'Mytilus'. We also thank Mariana Cueto, Félix Álvarez, Jose Antonio, Pilar Sieiro, Juan Abella and Sarah Jane Burke, who helped us with the technical analysis. We are indebted to Prof. Ángel Guerra for his valuable comments that improved the manuscript. This research was supported by CAIBEX (CTM-200766408-CO2-01), LARECO (CTM-2011-25929), CGL200763221, and CGL2012-39545. M.G. was funded by the Junta para la Ampliación de Estudios Predoctorales (CSIC) cofinanced with Fondo Social Europeo (ESF) funds.

\section{LITERATURE CITED}

> Akaike H (1974) A new look at the statistical model indentification. IEEE Trans Automat Contr 19:716-722

Amin OM, Heckmann RA, Van Ha NG (2011) Description of two new species of Rhadinorhynchus (Acanthocephala, Rhadinorhynchidae) from marine fish in Halong Bay, Vietnam, with a key to species. Acta Parasitol 56:67-77
Andrew NL, Mapstone BD (1987) Sampling and the description of spatial pattern in marine ecology. Oceanogr Mar Biol Annu Rev 25:39-90

Arai HP (1989) Acanthocephala. In: Margolis L, Kabata Z (eds) Guide to the parasites of fishes of Canada, Part III. Can Spec Publ Fish Aquat Sci, p 107-195

Braley M, Goldsworthy S, Page B, Steer M, Austin JJ (2010) Assessing morphological and DNA-based diet analysis techniques in a generalist predator, the arrow squid Nototodarus gouldi. Mol Ecol Resour 10:466-474

Bunkley-Williams L, Williams EH Jr (1996) Acanthocephala (spiny-headed worms) In: Parasites of offshore big game fishes of Puerto Rico and the Western Atlantic, San Juan and Mayaguez, Puerto Rico, Puerto Rico. Department of Natural and Environmental Resources and University of Puerto Rico, Mayaguez, p 156-162

Bush AO, Lafferty KD, Lotz JM, Shostak AW (1997) Parasitology meets ecology on its own terms: Margolis et al. revisited. J Parasitol 83:575-583

Bush AO, Fernández JC, Esch GW, Seed JR (2001) Acanthocephala: the thorny-headed worms. In: Bush AO, Fernández JC, Esch GW, Seed JR (eds) Parasitism: the diversity and ecology of animal parasites. Cambridge University Press, Cambridge, p 197-214

Cable RM, Linderoth J (1963) Taxonomy of some Acanthocephala from marine fishes with reference to species from Curaçao, N.A. and Jamaica, W.I. J Parasitol 49:706-716

> Castresana J (2000) Selection of conserved blocks from multiple alignments for their use in phylogenetic analysis. Mol Biol Evol 17:540-552

Chandler AC (1934) A revision of the genus Rhadinorhynchus (Acanthocephala) with descriptions of new genera and species. Parasitology 26:352-358

Costa G, Pontes T, Rego AA (2004) Prevalence, intensity and abundance of Rhadinorhynchus pristis (Acanthocephala, Rhadinorhynchidae) in chub mackerel Scomber japonicus (Pisces, Scombridae) from Madeira Island. Acta Parasitol 49:41-44

Deagle BE, Gales NJ, Evans K, Jarman SN, Robinson S, Trebilco R, Hindell M (2007) Studying seabird diet through genetic analysis of faeces: a case study on macaroni penguins (Eudyptes chrysolophus). PLoS ONE 2:e831

> García-Varela M, González-Oliver A (2008) The systematic position of Leptorhynchoides (Kostylew, 1924) and Pseudoleptorhynchoides (Salgado-Maldonado, 1976), inferred from nuclear and mitochondrial DNA gene sequences. J Parasitol 94:959-962

> García-Varela M, Nadler S (2005) Phylogenetic relationships of Palaeacanthocephala (Acanthocephala) inferred from SSU and LSU rDNA gene sequences. J Parasitol 91: 1401-1409

> García-Varela M, Nadler S (2006) Phylogenetic relationships among Syndermata inferred from nuclear and mitochondrial gene sequences. Mol Phylogenet Evol 40: $61-72$

> García-Varela M, Pérez-Ponce de León G, de la Torre P, Cummings MP, Sarma SSS, Laclette JP (2000) Phylogenetic relationships of Acanthocephala based on analysis of $18 \mathrm{~S}$ ribosomal RNA gene sequences. J Mol Evol 50: 532-540

> García-Varela M, Cummings MP, Pérez-Ponce de León G, Gardner SL, Laclette JP (2002) Phylogenetic analysis based on 18S ribosomal RNA gene sequence supports the existence of class Polyacanthocephala (Acanthocephala). Mol Phylogenet Evol 23:288-292 
Garey JR, Near TJ, Nonnemacher MR, Nadler SA (1996) Molecular evidence for Acanthocephala as a subtaxon of Rotifera. J Mol Evol 43:287-292

Golvan YJ (1969) Systématique des Acanthocéphales (Acanthocephala Rudolphi 1801). Première partie. L'ordre des Palaeacanthocephala Meyer 1931. Premier fascicule. La super-famille des Echinorhynchoidea (Cobbold 1876) Golvan et Houin 1963. Mém Mus Natn Hist Nat, Paris, Séries A, Zoologie, nouvelle série p 1-373

Gómez-Gutiérrez J, Robinson CJ, Kawaguchi S, Nicol S (2010) Parasite diversity of Nyctiphanes simplex and Nematoscelis difficilis (Crustacea: Euphausiacea) along the northwestern coast of Mexico. Dis Aquat Org 88: 249-266

> Gregori M, Aznar FJ, Abollo E, Roura A, González AF, Pascual S (2012) Nyctiphanes couchii as intermediate host for the acanthocephalan Bolbosoma balaenae in temperate waters of the NE Atlantic. Dis Aquat Org 99:37-47

Guisande C, Barreiro A, Maneiro I, Riveira I, Vergara A, Vaamonde A (2006) Tratamiento de datos. Ediciones Díaz de Santos, Madrid

> Herlyn H, Martini N, Ehlers U (2001) Organisation of the praesoma in Paratenuisentis ambiguus (Van Cleave, 1921) (Acanthocephala: Eoacanthocephala), with special reference to the lateral sense organs and the musculature. Syst Parasitol 50:105-116

> Herlyn H, Piskurek O, Schmitz J, Ehlers U, Zischler H (2003) The syndermatan phylogeny and the evolution of acanthocephalan endoparasitism as inferred from 18S rDNA sequences. Mol Phylogenet Evol 26:155-164

Hoberg EP, Daoust PY, McBurney S (1993) Bolbosoma capitatum and Bolbosoma sp. (Acanthocephala) from sperm whales (Physeter macrocephalus) stranded on Prince Edward Island, Canada. J Helminthol Soc Wash 60: 205-210

> Hogans WE, Brattey J, Uhazy LS, Hurley PC (1983) Helminth parasites of swordfish Xiphias gladius L. from the northwest Atlantic Ocean. J Parasitol 69:1178-1179

Katoh K, Misawa K, Kuma K, Miyata T (2002) MAFFT: a novel method for rapid multiple sequence alignment based on fast Fourier transform. Nucleic Acids Res 30: 3059-3066

Lindley JA (1977) Continuous plankton records: the distribution of the Euphausiacea (Crustacea: Malacostraca) in the North Atlantic and the North Sea, 1966-1967. J Biogeogr 4:121-133

Marcogliese DJ (1995) The role of zooplankton in the transmission of helminth parasites to fish. Rev Fish Biol Fish 5: 336-371

Marcogliese DJ (2002) Food webs and the transmission of parasites to marine fish. Parasitol 124:83-99

Mauchline J (1980) The biology of euphausiids. Adv Mar Biol 18:373-623

Mauchline J (1984) Euphausiid, stomatopod and leptostracan crustaceans. Key and notes for the identification of the species. In: Kermack DK, Barnes RSK (eds) Synopses of the British fauna (New Series, No 30). The Linnaean Society of London and the Estuarine and Brackish-Water Science Association, London, p 1-91

Miller DM, Dunagan TT (1985) Functional morphology. In: Crompton DWT, Nickol BB (eds) Biology of the Acanthocephala. Cambridge University Press, Cambridge, p 73-124

Monks S (2001) Phylogeny of the Acanthocephala based on morphological characters. Syst Parasitol 48:81-115
Near TJ (2002) Acanthocephalan phylogeny and the evolution of parasitism. Integr Comp Biol 42:668-677

- Near TJ, Garey JR, Nadler SA (1998) Phylogenetic relationships of the Acanthocephala inferred from 18S ribosomal DNA sequences. Mol Phylogenet Evol 10:287-298

Nickol BB (1985) Epizootiology. In: Crompton DWT, Nickol BB (eds) Biology of the Acanthocephala. Cambridge University Press, Cambridge, p 307-347

> Nickol BB, Heard RW Jr, Smith NF (2002) Acanthocephalans from crabs in the southeastern US, with the first intermediate hosts known for Arhythmorhynchus frassoni and Hexaglandula corynosoma. J Parasitol 88:79-83

Omori M, Ikeda T (1984) Methods in marine zooplankton ecology. John Wiley and Sons, New York, NY

Pascual S, González AF, Arias C, Guerra A (1996) Biotic relationships of Illex condetii and Todaropsis eblanae (Cephalopoda, Ommastrephidae) in the Northeast Atlantic: evidence from parasites. Sarsia 81:265-274

Pascual S, González AF, Guerra A (2007) Parasite recruitment and oceanographic regime: Evidence suggesting a relationship on a global scale. Biol Rev Camb Philos Soc 82:257-263

Petrochenko VI (1956) Acanthocephala of domestic and wild animals, Vol 1. Isdatel'stvo Akademii Nauk SSSR, Moscow (English Translation by the Israel Program for Scientific Translations, 1971)

Petrochenko VI (1958) Acanthocephala of domestic and wild animals, Vol 2. Isdatel'stvo Akademii Nauk SSSR, Moscow (English Translation by the Israel Program for Scientific Translations, 1971)

Pichelin S, Cribb TH (2001) The status of the Diplosentidae (Acanthocephala: Palaeacanthocephala) and a new family of acanthocephalans from Australia wrasses (Pisces: Labridae). Folia Parasitol 48:289-303

> Posada D (2008) ModelTest: phylogenetic model averaging. Mol Biol Evol 25:1253-1256

Rego AA (1987) Rhadinorhynchus pristis (Rudolphi, 1802) Acanthocephalan parasite of fishes, Scomber scombrus and $S$. japonicus. Some observations on the scanning electron microscope. Mem Inst Oswaldo Cruz 82: $287-288$

> Rego AA, Varela MC, Mendoça MM, Afonso-Roque MM (1985) Helmintofauna da sarda (Scomber scombrus L.) peixe da costa continental portuguesa. Mem Inst Oswaldo Cruz 80:97-100

> Reiczigel J (2003) Confidence intervals for the binomial parameter: some new considerations. Stat Med 22: 611-621

Rodrigues HO, Noronha D, Varela MC (1975) Alguns Acantocéfalos de peixes do Oceano Atlântico Costa continental portuguesa e costa do norte da África. Mem Inst Oswaldo Cruz 73:209-214

Roura A, González AF, Redd K, Guerra A (2012) Molecular prey identification in wild Octopus vulgaris paralarvae. Mar Biol 159:1335-1345

Roura A, Álvarez-Salgado XA, González AF, Gregori M, Rosón G, Guerra A (2013) Short-term meso-scale variability of mesozooplankton communities in a coastal upwelling system (NW Spain). Prog Oceanogr 109: $18-32$

> Rózsa L, Reiczigel J, Majoros G (2000) Quantifying parasites in samples of host. J Parasitol 86:228-232

Sars GO (1885) The voyage of H.M.S. Challenger. Zoology. Report on the Schizopoda collected by H.M.S. Challenger during the Years 1873-76. Vol 13. Edinburgh 
Shimazu T (1975) Some cestode and acanthocephalan larvae from euphausiid crustaceans collected in the northern North Pacific Ocean. Bull Jpn Soc Sci Fish 41:813-821

Suzuki N, Hoshino K, Murakami K, Takeyama H, Chow S (2008) Molecular diet analysis of phyllosoma larvae of the Japanese spiny lobster Palinurus japonicus (Decapoda: Crustacea). Mar Biotechnol 10:49-55

Tamura K, Peterson D, Peterson N, Stecher G, Nei M, Kumar S (2011) MEGA5: Molecular evolutionary genetics analysis using maximum likelihood, evolutionary distance, and maximum parsimony methods. Mol Biol Evol 28: 2731-2739

Taraschewski H (2005) Acanthocephala (thorny or spinyheaded worms). In: Rohde K (ed) Marine parasitology. CABI Publishing, Wallingford, p 116-121

Tavaré S (1986) Some probabilistic and statistical problems in the analysis of DNA sequences. In: Miura RM (ed) Some mathematical questions in biology: DNA sequence analysis. American Mathematical Society, Providence, RI, p 57-86

Troncy PM, Vassiliades G (1973) Acanthocephalan parasites of fish in Africa. Bull. IFAN Ser A Sci Nat 35:522-539

Editorial responsibility: David Marcogliese, Montreal, Quebec, Canada
Tsimbalyuk EM (1980) Results of an examination of the parasite fauna of planktonic organisms in far-eastern seas of the USSR. Voprosy parazitologii vodnykh bespozvonochnykh zhivotnykh (Tematicheskii Sbornik). Akademiya Nauk Litovskoi SSR, Institut Zoologii i Parazitologii, Vilnyus, p 103-104 (in Russian)

Vassiliades G (1982) Helminthes parasites des poissons de mer des côtes du Senegal. Bull IFAN 44:78-99

> Verweyen L, Klimpel S, Palm HW (2011) Molecular phylogeny of the Acanthocephala (Class Palaeacanthocephala) with a paraphyletic assemblage of the orders Polymorphida and Echinorhynchida. PLoS ONE 6: e28285

Yamaguti S (1963) Systema Helminthum, Vol 5. Acanthocephala. Wiley Interscience, New York, NY

Zdzitowiecki K (1989) New data on the morphology and distribution of two acanthocephalans, Andracantha baylisi (Zdzitowiecki, 1986) comb. n. and Corynosoma australe Johnston, 1937. Acta Parasitol Pol 34:167-172

Zdzitowiecki K (1991) Antarctic Acanthocephala. In: Wägele JW, Sieg J (eds) Synopses of the Antarctic benthos, Vol 3. Koeltz Scientific Books, Koenigstein, p 1-116

Submitted: August 6, 2012; Accepted: April 23, 2013

Proofs received from author(s): June 27, 2013 